$12-2009$

\title{
Paroxysmal atrial fibrillation presenting as anterior wall STEMI in an elderly woman
}

Muhammad Asim Shabbir

Muhammad Hamza Saad Shaukat

Lance Sullenberge

Mikhail Torosof

Follow this and additional works at: https://ecommons.aku.edu/pakistan_fhs_mc_med_intern_med

Part of the Internal Medicine Commons 
Case report

\title{
Paroxysmal atrial fibrillation presenting as anterior wall STEMI in an elderly woman
}

\author{
Muhammad Asim Shabbir, ${ }^{1}$ Muhammad Hamza Saad Shaukat, ${ }^{\oplus 1,2}$ \\ Lance Sullenberger, ${ }^{3}$ Mikhail Torosoff $^{4}$
}

'Internal Medicine, Albany Medical Center Hospital, Albany, New York, USA

${ }^{2}$ Aga Khan University, Karachi, Pakistan

${ }^{3}$ Capital Cardiology Associates, Albany Medical Center Hospital, Albany, New York, USA

${ }^{4}$ Cardiology, Albany Medical Center Hospital, Albany, New York, USA

Correspondence to

Dr Muhammad Hamza Saad Shaukat,

hamzasaad1991@gmail.com

Accepted 23 August 2019

Check for updates

(c) BMJ Publishing Group Limited 2019. No commercial re-use. See rights and permissions. Published by BMJ.

To cite: Shabbir MA

Saad Shaukat MH,

Sullenberger $\mathrm{L}$, et al. BMJ

Case Rep 2019:12:e231996.

doi:10.1136/bcr-2019-

231996

\section{SUMMARY}

A 77-year-old woman without traditional risk factors for coronary artery disease (CAD) underwent coronary CTangiography for evaluation of palpitations after negative Holter monitoring and non-diagnostic ECG exercise stress test. Coronary artery calcium score was reported zero; 1 day later, she was admitted with anteriorwall ST elevation myocardial infarction. Acute left anterior descending artery thrombus was treated with mechanical thrombectomy and Percutaneous Coronary Intervention ( $\mathrm{PCI})$. Interestingly, the coronary arteries were angiographically normal. During hospitalisation, paroxysmal atrial fibrillation was noted followed by initiation of anticoagulation. Echocardiogram did not show thrombus or atrial shunt. Cardioversion with Sotalol was successful. Myocardial infraction was most likely cardioembolic secondary to paroxysmal atrial fibrillation-consistent with longstanding history of palpitations. Accounting for $3 \%$ of acute coronary syndromes, coronary embolism is treated with therapeutic anticoagulation for at least 3 months irrespective of cause and carries a higher risk of adverse cardiovascular events.

\section{BACKGROUND}

Estimated 3\% of acute coronary syndromes (ACS) are caused by coronary embolism (CE). In patients with ACS without significant underlying coronary artery disease (CAD), embolic aetiology should be considered. Diagnosis of CE may be difficult in patients with paroxysmal atrial fibrillation, as the rhythm may be normal at presentation. Identification of CE subgroup of patients with ACS is important because of increased risk for major adverse cardiac outcomes and importance of systemic anticoagulation in prevention of future embolic events.

\section{CASE PRESENTATION}

A 77-year-old non-smoker woman presented with sudden onset substernal chest pain radiating to the left arm. ECG showed anterior wall ST-elevation myocardial infarction (STEMI). Haemodynamically stable, emergent cardiac catheterisation revealed a $100 \%$ acute occlusion of the proximal left anterior descending artery (LAD, figure $1 \mathrm{~A})$. Left main, circumflex and right coronary arteries were normal (0\% stenosis reported); no circumflex-LAD or right coronary artery (RCA)-LAD collaterals were noted. Mechanical thrombectomy was performed followed by the placement of two stents in the proximal LAD. Guideline-directed post-STEMI medical therapy was initiated: aspirin, clopidogrel, beta-adrenergic antagonist, statin and ACE inhibitor.

Few weeks prior to her presentation with STEMI, the patient was seen in outpatient cardiology office for daily symptoms of palpitations and progressive exertional dyspnoea. According to the patient, her heartbeat has 'felt irregular' for several years. No prior history of hypertension, diabetes mellitus, dyslipidemia, atrial fibrillation or cardiovascular disease was reported. At the office visit, 12-lead ECG was sinus rhythm with premature ventricular complexes (PVCs). 24 hours Holter monitor was notable for sinus rhythm with premature atrial contractions $(2.1 \%$ of beats) and PVCs $(1.2 \%$ of beats). The patient then underwent an ECG stress test with non-diagnostic ST and T wave changes.

Given the non-diagnostic stress test and atypical symptoms, 1 day prior to her presentation with STEMI, a coronary CT angiogram was performed which revealed a coronary artery calcium (CAC) score of zero and no CAD whatsoever.

\section{INVESTIGATIONS}

1. Troponin $10.38 \mathrm{ng} / \mathrm{mL}$ (reference: $<0.05 \mathrm{ng}$ ) $\mathrm{mL})$.

2. Fasting lipid panel-low density lipoprotein (LDL) $118 \mathrm{mg} / \mathrm{dL}$; total cholesterol $202 \mathrm{mg} / \mathrm{dL}$ (reference: $<200 \mathrm{mg} / \mathrm{dL}$ ); triglycerides $100 \mathrm{mg}$ / $\mathrm{dL}$ (reference: $<150 \mathrm{mg} / \mathrm{dL}$ ); high density lipoprotein (HDL) $64 \mathrm{mg} / \mathrm{dL}$ (reference: $>35 \mathrm{mg}$ / dL).

3. Fasting serum glucose $100 \mathrm{mg} / \mathrm{dL}$.

4. Transthoracic ECHO: Left ventricular ejection fraction 35\%-39\% with segmental wall motion abnormalities. Normal-sized left and right atria. No mitral stenosis or aortic valve abnormality. No patent foramen ovale.

5. Echocardiographic evaluation was negative for an inter-atrial communication or cardiac masses.

6. No inter-atrial communication was detected by the right heart catheterisation.

\section{DIFFERENTIAL DIAGNOSIS}

Given the normal coronary arteries on computed tomography angiography (CTA) followed by LAD occlusion with high clot burden, investigation for non-atherosclerotic embolic causes ensued. There was no history of hypercoagulable state, and the 


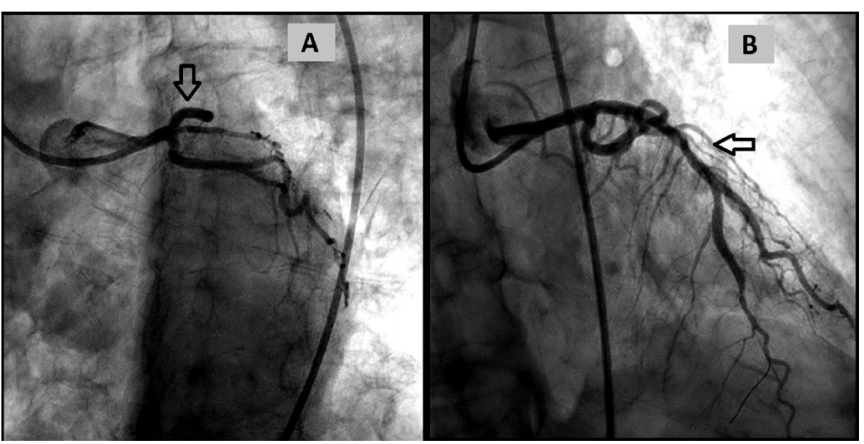

Figure 1 (A) Left anterior oblique (LAO) caudal view of proximal left anterior descending artery (LAD) occlusion (arrow). (B) Right anterior oblique (RAO) cranial view of non-diseased LAD (arrow) postthrombectomy and Percutaneous Coronary Intervention (PCI).

patient denied miscarriages or arterial/venous thrombosis. Likewise, no history of atrial septal defect, endocarditis or recent/ remote cardiac intervention was reported. Transthoracic echocardiogram was unrevealing for an intracardiac shunt, thrombus or mass. Despite extensive discussion and explanation of the diagnostic and therapeutic benefit, the patient declined transoesophageal echocardiography. Extended ambulatory rhythm monitoring was planned but on the second day of hospitalisation, paroxysmal atrial fibrillation was recorded on telemetry (figure 2).

\section{TREATMENT}

Systemic oral anticoagulation was initiated (Apixaban $5 \mathrm{mg}$ two times per day) followed by successful pharmacologic cardioversion with Sotalol. Dual antiplatelet (aspirin and clopidogrel) and post-STEMI guideline-directed medical therapy were continued, including high intensity statin and ACE inhibitor, but metoprolol was replaced by Sotalol.

\section{OUTCOME AND FOLLOW-UP}

The patient was discharged after unremarkable 72 hours monitoring for QTc prolongation post-initiation of Sotalol. Triple therapy with aspirin, clopidogrel and apixaban was continued at discharge with the intent to discontinue aspirin after 6 weeks to minimise bleeding risk. No chest pain, exertional dyspnoea or heart failure symptoms were reported at 1 week follow-up. The patient's ECG showed normal sinus rhythm with resolving ST-elevations. Ejection fraction was preserved on repeat transthoracic ECHO. Monthly appointments are scheduled for close monitoring and observation for bleeding on long-term anticoagulation $($ CHA2DS2VASc score $=5$ ) and clopidogrel.

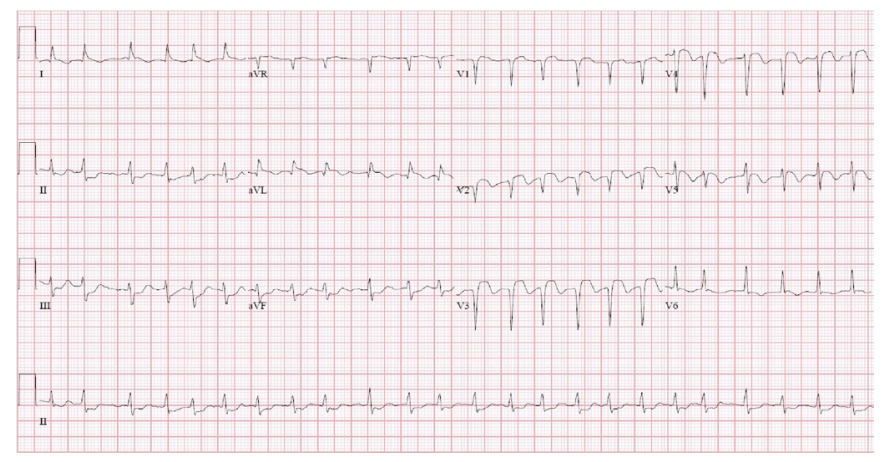

Figure 2 Atrial fibrillation on 12-lead ECG.

\section{DISCUSSION}

Causes of CE are broadly divided into three categories: direct, paradoxical and iatrogenic. ${ }^{1}$ In causes classified as direct, the embolus originates from a thrombus in the left atrium and ventricle, mitral and aortic valves, pulmonary veins or proximal coronary artery - with paroxysmal or persistent atrial fibrillation being the most common cause $(73 \%))^{2}$ Other direct aetiologies of CE due to the left-sided thrombus include endocarditis and post-myocardial infraction left ventricular thrombus. ${ }^{1}$ Paradoxical CE may also be secondary to venous thrombi entering the systemic circulation through an atrial septal defect, patent foramen ovale or pulmonary arteriovenous malformation. ${ }^{1}$

When compared with the cerebral and systemic circulation, the coronary arterial vasculature is relatively protected from emboli because of difference in calibre of the aorta and main coronary arteries, the acute angle at which the coronaries originate from the aorta, and the fast flow across the coronary ostia. ${ }^{1}$ Consequently, CE is a rare cause of ACS overall (2.9\%) and STEMI $(4.3 \%)^{23}$

Clinical presentation of acute myocardial infarction due to $\mathrm{CE}$ is indistinguishable from atherosclerotic myocardial infarction. ${ }^{1}$ Consequently, the initial ACS management is the same, regardless of the aetiology. However, recognition of CE, in addition to specific medical management, is important because of the need for closer monitoring in this subpopulation and increased risk of cardiac death as compared with the patients with atherosclerotic myocardial infarction. ${ }^{3}$ Patients with CE myocardial infarction suffer from significantly increased 5 -year risk of adverse cardiac and cerebrovascular events, which may be as high as $27 \% .^{2}$ Age greater than 60 years, female gender, reduced left ventricular ejection fraction and atrial fibrillation are independent risk factors for major adverse cardiovascular events in patients with non-atherosclerotic myocardial infarction, ${ }^{4}$ emphasising the importance of recognising CE and associated prognostic factors.

$\mathrm{CE}$ diagnosis may be suspected on retrospective evaluation of coronary angiogram when the occlusive thrombus burden is disproportionate to the degree of atherosclerosis, no or minimal atherosclerosis in other coronary territories, or there is evidence of concomitant systemic embolisation in other arterial beds, for example, cerebral, mesenteric, retinal or in extremities. ${ }^{2} \mathrm{Com}-$ pared with those patients with atherosclerotic CAD, patients with $\mathrm{CE}$ are less likely to have traditional risk factors-hypertension, dyslipidemia, smoking and diabetes. ${ }^{5}$ However, it may reflect a selection bias, as more thorough search for embolic source is performed in patients with ACS without significant risk factors for systemic atherosclerosis. Anticoagulation is the treatment of choice for all patients with CE, and thrombophilia testing is not routinely indicated. ${ }^{1}$ However, decision for testing needs to be individualised, for instance to determine duration of treatment in patients with unexplained venous thromboembolism and/or who are at higher risk for bleeding.

Major CE diagnostic criteria (suggested by Shibata et $a l^{2}$ ) include (1) angiographic findings of coronary vessel occlusion without atherosclerosis; (2) concomitant involvement of multiple coronary territories and/or distal systemic embolism; (3) histologic evidence of venous thrombus and (4) echocardiographic/ $\mathrm{CT} / \mathrm{MRI}$ evidence of intracardiac thrombus. Minor criteria include (1) $<25 \%$ stenosis of non-culprit coronary vessels, (2) known atrial fibrillation and (3) risk factor for embolism (eg, patent foramen ovale, atrial septal defect, dilated cardiomyopathy, prosthetic valve, etc.). Our patient met one major criterion (angiographic evidence of large thrombus without significant underlying atherosclerosis, figure 1B) and two minor criteria 
(atrial fibrillation and $<25 \%$ stenosis in non-culprit vessels) qualifying as definite $\mathrm{CE} .^{2}$

There was no significant coronary atherosclerosis by invasive angiogram in the acute setting. Moreover, a coronary CTA performed just 1 day prior to the STEMI presentation did not reveal any evidence for atherosclerotic disease whatsoever. False-negative results of CT coronary angiography do occur with reported negative predictive values of $80 \%-90 \%{ }^{6}$ However, these false-negative results typically occur in patients with atherosclerotic disease in whom a particular intracoronary lesion is 'undercalled' by the reader. In the case presented, the CAC score of 0 and finding of no non-calcified plaque make a false-negative CT result very unlikely. Finally, in some patients, myocardial infarction may cause atrial fibrillation. ${ }^{7}$ But our patient had longstanding history of palpitations which, in retrospect, were probably due to undiagnosed paroxysmal atrial fibrillation. In suspected embolic infarct, post-thrombectomy intravascular ultrasound would have been useful in ruling out dissection or eroded plaque and, in the presence of normal vessel morphology, would have confirmed the diagnosis of embolism. ${ }^{8}$ In confirmed embolic myocardial infarction, it would have obviated the need for triple antithrombotic therapy (dual antiplatelet and anticoagulation).

The chronology of events in the presented case serves as a reminder of the clinical characteristics associated with CE, its diagnostic evaluation and treatment to reduce risk of recurrence. Although there are no formal guidelines for management of CE, workup should include extended cardiac rhythm monitoring for detection of paroxysmal or persistent atrial fibrillation (the most common cause of CE) and transoesophageal echocardiography for detection of left atrial thrombus and right-to-left shunt. In retrospect, delayed enhancement cardiac MRI could have been offered as an alternative to transoesophageal echocardiography for left atrial thrombus detection. ${ }^{9}$

The recommended treatment for CE is systemic anticoagulation. The duration of anticoagulation in CE due to paradoxical right-to-left embolism depends on the underlying thrombosis aetiology and risk-profile, with treatment duration of 3 months if risk factors are transient, or longer if persistent. ${ }^{1}$ As in the presented patient, if atrial fibrillation is detected (CHA2DS2 VASc assigned to a minimum of two in men and three in women with CE), long-term anticoagulation is indicated. Additional complexity of patient care and increased bleeding risk due to dual antiplatelet therapy and systemic anticoagulation should be considered as well. In patients with increased bleeding risk on dual antiplatelet therapy who require systemic anticoagulation, aspirin may be stopped 4 weeks after the acute event with acceptable long-term outcomes. ${ }^{7}$ Thrombectomy and longterm anticoagulation are adequate therapy for CE secondary to atrial fibrillation. The role of left atrial appendage closure for prevention of the CE events in patients with atrial fibrillation is currently unclear. This case highlights the benefit of evaluating suspected CE with intravascular ultrasound or optical coherence tomography post-thrombectomy to avoid unnecessary percutaneous coronary intervention and bleeding risk associated with triple antithrombotic therapy.

\section{Learning points}

- Coronary embolism (CE) is an under-recognised cause of acute coronary syndrome associated with worse cardiac and cerebrovascular outcomes compared with atherosclerotic heart disease.

- Atrial fibrillation is the most common cause of CE. Longterm rhythm monitoring for detection of atrial fibrillation is indicated.

- Thrombophilia testing is not routinely indicated in CE except for unexplained venous thrombosis.

- Increased bleeding risk due to dual antiplatelet therapy and systemic anticoagulation should be considered. Evaluation of suspected CE with post-thrombectomy intravascular ultrasound may prevent unnecessary percutaneous coronary intervention and bleeding risk associated with triple antithrombotic therapy.

Contributors MHSS and MAS drafted the manuscript. MT and LS helped with editing and literature review.

Funding The authors have not declared a specific grant for this research from any funding agency in the public, commercial or not-for-profit sectors.

Competing interests None declared.

Patient consent for publication Obtained.

Provenance and peer review Not commissioned; externally peer reviewed.

\section{REFERENCES}

1 Raphael CE, Heit JA, Reeder GS, et al. Coronary Embolus: An Underappreciated Cause of Acute Coronary Syndromes. JACC Cardiovasc Interv 2018;11:172-80.

2 Shibata T, Kawakami S, Noguchi T, et al. Prevalence, clinical features, and prognosis of acute myocardial infarction attributable to coronary artery embolism. Circulation 2015:132:241-50.

3 Popovic B, Agrinier N, Bouchahda N, et al. Coronary embolism among ST-segmentelevation myocardial infarction patients. Circulation: Cardiovascular Interventions 2018;11:e005587

4 Abdu FA, Liu L, Mohammed AQ, et al. Myocardial infarction with non-obstructive coronary arteries (MINOCA) in Chinese patients: Clinical features, treatment and 1year follow-up. Int J Cardiol 2019.

5 Kolodgie FD, Virmani R, Finn AV, et al. Embolic myocardial infarction as a consequence of atrial fibrillation. Circulation 2015;132:223-6.

6 Miller JM, Rochitte CE, Dewey M, et al. Diagnostic performance of coronary angiography by 64-row CT. N Eng/ J Med 2008;359:2324-36.

7 January CT, Wann LS, Alpert JS, et al. 2014 AHA/ACC/HRS guideline for the management of patients with atrial fibrillation: a report of the American College of Cardiology/American Heart Association Task Force on Practice Guidelines and the Heart Rhythm Society. J Am Coll Cardiol 2014;64:e1-76.

8 Reynolds HR. Myocardial infarction without obstructive coronary artery disease. Curr Opin Cardiol 2012;27:655-60.

9 Chen J, Zhang H, Zhu D, et al. Cardiac MRI for detecting left atrial/left atrial appendage thrombus in patients with atrial fibrillation : Meta-analysis and systematic review. Herz 2019;44. 
Copyright 2019 BMJ Publishing Group. All rights reserved. For permission to reuse any of this content visit https://www.bmj.com/company/products-services/rights-and-licensing/permissions/

BMJ Case Report Fellows may re-use this article for personal use and teaching without any further permission.

Become a Fellow of BMJ Case Reports today and you can:

- Submit as many cases as you like

- Enjoy fast sympathetic peer review and rapid publication of accepted articles

- Access all the published articles

- Re-use any of the published material for personal use and teaching without further permission

Customer Service

If you have any further queries about your subscription, please contact our customer services team on +44 (0) 2071111105 or via email at support@bmj.com.

Visit casereports.bmj.com for more articles like this and to become a Fellow 